PROCEEDINGS OF THE

AMERICAN MATHEMATICAL SOCIETY

Volume 127, Number 2, February 1999, Pages 337-341

S 0002-9939(99)04684-5

\title{
GROUP ALGEBRAS WITH UNITS SATISFYING A GROUP IDENTITY II
}

\author{
CHIA-HSIN LIU AND D. S. PASSMAN
}

(Communicated by Ken Goodearl)

\begin{abstract}
We classify group algebras of torsion groups over a field of characteristic $p>0$ with units satisfying a group identity.
\end{abstract}

\section{INTRODUCTION}

A group $U$ is said to satisfy a group identity if there exists a nontrivial word $w=$ $w\left(x_{1}, \ldots, x_{n}\right)$ in the free group generated by $x_{1}, \ldots, x_{n}$ such that $w\left(u_{1}, \ldots, u_{n}\right)=$ 1 for all $u_{i} \in U$. In early 1980s, Brian Hartley made the conjecture that if the units of the group algebra of a torsion group $G$ over a field $K$ satisfy a group identity, then the group algebra $K[G]$ satisfies a polynomial identity. This was settled recently for group algebras over infinite fields in [GSV97], and completely solved in [Liu]. Some natural questions we can ask are: "If the group algebra satisfies a polynomial identity, does the unit group satisfy a group identity? If not, what additional conditions are required to make it true?" After [GSV97] appeared, these questions were answered in [Pas97] for group algebras over infinite fields. Indeed, the paper showed that, for the group algebra $K[G]$ of a torsion group $G$ over an infinite field $K$ of characteristic $p>0$, the unit group satisfies a group identity if and only if $K[G]$ satisfies a polynomial identity and $G^{\prime}$ is a $p$-group of bounded period. The proof given in [Pas97] uses two facts: [GJV94, Proposition 1] and [GSV97, Lemma 2.3]. [GJV94, Proposition 1] basically says that if units of an algebra over an infinite field satisfy a group identity, then the product of any two square zero elements is nilpotent of bounded degree. This proposition was modified and extended to algebras over an arbitrary field in [Liu, Lemmas 3.1, 3.2], and thus it is natural to expect that the results in [Pas97] can be extended to group algebras over finite fields. On the other hand, [GSV97, Lemma 2.3] asserts that for any nonabelian finite group $G$ and any infinite field $K$ of characteristic $p>0$, if the units of the group algebra $K[G]$ satisfy a group identity, then $G^{\prime}$ must be a finite $p$-group. This is no longer true when $K$ is finite. Actually, if $G^{\prime}$ is a $p$-group, then we do obtain the same result as in [Pas97].

Theorem 1.1. Let $K[G]$ be the group algebra of a torsion group $G$ over a field $K$ of characteristic $p>0$ and let $U(K[G])$ be the group of units of $K[G]$. If $G^{\prime}$ is a $p$-group, then the following are equivalent.

Received by the editors May 15, 1997.

1991 Mathematics Subject Classification. Primary 16S34.

Key words and phrases. Group algebras, units, group identities.

(C)1999 American Mathematical Society 
1. $U(K[G])$ satisfies a group identity.

2. G has a normal p-abelian subgroup of finite index, and $G^{\prime}$ has bounded period.

3. $U(K[G])$ satisfies $(x, y)^{p^{k}}=1$ for some integer $k \geq 0$.

Surprisingly, if $G^{\prime}$ is not a $p$-group, then not only can the period of $G^{\prime}$ be bounded, but also the period of the whole group $G$ can be bounded.

Theorem 1.2. Let $K[G]$ be the group algebra of a torsion group $G$ over a field $K$ of characteristic $p>0$ and let $U(K[G])$ be the group of units of $K[G]$. If $G^{\prime}$ is not a p-group, then the following are equivalent.

1. $U(K[G])$ satisfies a group identity.

2. $G$ has a normal p-abelian subgroup of finite index, $G$ has bounded period and $K$ is finite.

3. $U(K[G])$ satisfies $x^{n}=1$ for some integer $n$.

\section{Proofs of the theorems}

The implications $3 \Rightarrow 1$ are trivial. The implication $2 \Rightarrow 3$ in Theorem 1.1 has been proved by [Pas97, Section 3] whether the field $K$ is infinite or finite. The implication $2 \Rightarrow 3$ in Theorem 1.2 can be obtained from the proof of [Coe82, Theorem A]. So we need to prove $1 \Rightarrow 2$ in both theorems.

We assume that $G$ is a torsion group and that $K$ is a field of characteristic $p>0$. Also, we assume that the group of units $U(K[G])$ of the group algebra $K[G]$ satisfies the group identity $w=1$. In view of [Liu, Theorem 1.1] and [Pas85, Corollary 5.3.10], $G$ has a normal $p$-abelian subgroup $A$ of finite index. In particular, $G$ is locally finite.

Let us record some lemmas we need. The following is from [Liu, Lemma 2.3].

Lemma 2.1. Let $R=K[H]$ be the group algebra of a locally finite group $H$ and assume that the group of units $U(R)$ satisfies $w=1$. If $S$ is any subalgebra of $R$ or $\bar{R}$ is any homomorphic image of $R$, then $U(S)$ and $U(\bar{R})$ also satisfy $w=1$.

The following lemma is from [Liu, Lemma 3.2]. Note that this result is an analogue of [GJV94, Proposition 1] for algebras over arbitrary fields and plays a crucial role in our proofs.

Lemma 2.2. Let $R$ be an algebra over a field $K$ and suppose $U(R)$ satisfies $w=1$. Let $a, b \in R$ such that $a^{2}=b^{2}=0$. If $a b$ is nilpotent, then $(a b)^{d}=0$ for some integer d determined by $w$.

For the rest of the paper, we fix notation so that $d$ will be as in the above lemma. If $M_{n}(F)$ is the $n$ by $n$ matrix algebra over a field $F$ and $U\left(M_{n}(F)\right)$ satisfies $w=1$, then we have the following bounds on the size of the field and the degree $n$ as shown in [Liu, Lemma 3.3].

Lemma 2.3. Let $F$ be any field. If $U\left(M_{n}(F)\right)$ satisfies $w=1$ and $n \geq 2$, then

1. $|F| \leq d$ and hence $F$ is a finite field.

2. $n<2 \log _{|F|} d+2 \leq 2 \log _{2} d+2$.

Let $m$ be the smallest integer not less than $2 \log _{2} d+2$ and define

$$
N=\prod_{|F| \leq d}\left|U\left(M_{m}(F)\right)\right| .
$$

Certainly, $N$ is finite and determined by $d$. 
Lemma 2.4. Let $x$ be a nonidentity $p^{\prime}$-element in $G^{\prime}$, and let $y$ be a nonidentity $p^{\prime}$-element in a normal $p^{\prime}$-subgroup of $G$. If $U(K[G])$ satisfies $w=1$, then $y^{N}=1$.

Proof. Suppose by way of contradiction that $y^{N} \neq 1$. Since $x \in G^{\prime}$, we can write

$$
x=\left(x_{1}, y_{1}\right)\left(x_{2}, y_{2}\right) \cdots\left(x_{n}, y_{n}\right) \neq 1 .
$$

Note that $x^{-1} y^{N}$ is a $p^{\prime}$-element since $y$ is in a normal $p^{\prime}$-subgroup of $G$. If $x \neq y^{N}$, let $\alpha=\left(1-x^{-1}\right)\left(1-y^{N}\right)$, then $\alpha$ is not a nilpotent by [Pas85, Lemma 2.3.3]. If $x=y^{N}$, let $\alpha=1-x$, so that $\alpha$ is also not nilpotent in this case. Observe that $H=\left\langle x_{1}, y_{1}, \ldots, x_{n}, y_{n}, y\right\rangle$ is a finite subgroup of $G$ since $G$ is locally finite. If $K$ is infinite, then $G^{\prime}$ is a $p$-group by [Pas97, Theorem 1.1]. Thus $K$ is finite here. Let $J=J(K[H])$, the Jacobson radical of $K[H]$, and now write $K[H] / J=$ $\bigoplus \sum_{i} M_{n_{i}}\left(F_{i}\right)$ where the $F_{i}$ are fields since $K$ is finite. Now $\alpha$ is not nilpotent, so $\alpha+J$ is not zero in $K[H] / J$. Hence there exists a natural map

$$
\theta: K[H] / J \rightarrow M_{n_{j}}\left(F_{j}\right)
$$

for some $j$ with $\theta(\alpha+J) \neq 0$. In particular, $\theta\left(1-x^{-1}+J\right) \neq 0$ and $\theta(1-x+J) \neq 0$. If $n_{j}=1$, then

$$
\theta(x+J)=\prod_{i=1}^{n} \theta\left(\left(x_{i}, y_{i}\right)+J\right)=\prod_{i=1}^{n}\left(\theta\left(x_{i}+J\right), \theta\left(y_{i}+J\right)\right)=1
$$

since $F_{j}$ is commutative. But $\theta\left(1-x^{-1}+J\right) \neq 0$, and hence $n_{j} \geq 2$. Also $U\left(M_{n_{j}}\left(F_{j}\right)\right)$ satisfies $w=1$ by Lemma 2.1. Hence $n_{j} \leq m$ and $\left|F_{j}\right| \leq d$ by Lemma 2.3. So we get $\theta\left(y^{N}+J\right)=\theta(y+J)^{N}=1$ since $\theta(y+J) \in U\left(M_{n_{j}}\left(F_{j}\right)\right) \hookrightarrow$ $U\left(M_{m}\left(F_{j}\right)\right)$. This implies that $\theta(\alpha+J)=0$, a contradiction. Therefore, $y^{N}=$ 1.

The following is an analogue of [Pas97, Lemma 2.3].

Lemma 2.5. Suppose that $G=\langle A, t\rangle$ where $A$ is a normal abelian p-subgroup and $t$ has finite order $q$. If $U(K[G])$ satisfies $w=1$, then $G^{\prime}$ has finite period.

Proof. The proof given in [Pas97, Lemma 2.3] basically works here. First, [Pas97, Lemma 2.1] holds for group algebras over arbitrary fields by Lemma 2.1. The argument given in the proof of [Pas97, Lemma 2.3] shows that we can assume $G$ is the semidirect product of $A$ by $\langle t\rangle$ and that $t$ has prime order $q$. So the only concern now is how we use Lemma 2.2, an analogue of [GJV94, Proposition 1].

If $q \neq p$, we take two square zero elements $\alpha=\tau a^{-1}\left(1-t^{-1}\right)$ and $\beta=$ $(q a-\operatorname{tr}(a)) \tau$ as in the proof of [Pas97, Lemma 2.3]. Notice that $q a-\operatorname{tr}(a)$ has augmentation 0 hence is in the augmentation ideal $\omega(K[A])$. But now $A$ is a locally finite normal $p$-subgroup of $G$ of finite index, so we have $\omega(K[A])=J(K[A])$ and $J(K[A]) K[G] \subseteq J(K[G])$ by [Pas85, Lemma 8.1.17] and [Pas85, Theorem 7.2.7]. This implies that $\beta$ and hence $\alpha \beta$ are in $J(K[G])$. Also, $J(K[G])$ is nil since $G$ is locally finite and we see that $\alpha \beta$ is nilpotent. Therefore, we can apply Lemma 2.2 to conclude that $(\alpha \beta)^{d}=0$ for some integer $d$ depending on the group identity.

If $q=p$, both $\tau$ and $a^{-1} \tau a$ have square 0 and augmentation 0 , so the product $\tau a^{-1} \tau a$ is in $\omega(K[G])$. But now $G$ is a locally finite $p$-group, so $\omega(K[G])$ is nil and Lemma 2.2 implies that $\left(\tau a^{-1} \tau a\right)^{d}=0$.

Therefore, the proof of [Pas97, Lemma 2.3] applies here and we deduce that $G^{\prime}$ has finite period. 
Lemma 2.6. Suppose that $A$ is a normal abelian p-subgroup of $G$ of finite index. If $U(K[G])$ satisfies $w=1$, then $G^{\prime}$ has finite period.

Proof. Use Lemma 2.5 and the proof of [Pas97, Lemma 2.4].

Lemma 2.7. If $U(K[G])$ satisfies $w=1$ and $G^{\prime}$ is not a p-group, then the $p^{\prime}$ elements of $G$ have finite period.

Proof. Since $U(K[G])$ satisfies $w=1$, [Liu, Theorem 1.1] and [Pas85, Corollary 5.3.10] imply that $G$ has a normal $p$-abelian subgroup $A$ of finite index. Note that $A^{\prime}$ is a finite normal $p$-subgroup of $G,\left(G / A^{\prime}\right)^{\prime}$ is not a $p$-group, and $U\left(K\left[G / A^{\prime}\right]\right)$ satisfies $w=1$ by Lemma 2.1. Thus it suffices to consider $G / A^{\prime}$, or equivalently, we may assume that $A$ is abelian. Write $A=P \times Q$ where $P$ is the set of $p$-elements of $A$ and $Q$ is the set of $p^{\prime}$-elements of $A$. Since $A$ is a normal abelian subgroup of $G, P$ and $Q$ are normal subgroups of $G$. Also, $A$ is a subgroup of $G$ of finite index, so it suffices to bound the period of $Q$. Now since $G^{\prime}$ is not a $p$-group, there exists a nonidentity $p^{\prime}$-element $x$ in $G^{\prime}$. For any nonidentity $y$ in $Q$, we have $y^{N}=1$ by Lemma 2.4. This shows that $Q$ has finite period and hence the $p^{\prime}$-elements of $G$ have finite period.

Lemma 2.8. If $U(K[G])$ satisfies $w=1$, then $G^{\prime}$ has finite period.

Proof. As in the proof of Lemma 2.7, we can assume that $A$ is abelian and write $A=P \times Q$. If $G^{\prime}$ is a $p$-group, it suffices to consider $G / Q$ since $Q$ is a $p^{\prime}$-group. If $G^{\prime}$ is not a $p$-group, Lemma 2.7 implies that $Q$ has finite period, hence it still suffices to consider $G / Q$ in this case. We can now assume that $A$ is a $p$-group. Therefore $G^{\prime}$ has finite period by Lemma 2.6.

Lemma 2.9. If $U(K[G])$ satisfies $w=1$ and $G^{\prime}$ is not a p-group, then the $p$ elements of $G$ have bounded period.

Proof. As usual, we can assume that $A$ is abelian and write $A=P \times Q$. If $B=$ $(P, G)$, then $B$ is a normal subgroup of $G$ contained in $P \cap G^{\prime}$. Thus $B$ is a $p$ group of finite period by Lemma 2.8. Therefore, it suffices to consider $G / B$, or equivalently we can assume that $P$ is central in $G$. Now, notice that $A$ has finite index in $G$, hence it suffices to bound the period of $P$.

Since $G^{\prime}$ is not a $p$-group, we can find a $p^{\prime}$-element in $G^{\prime}$ with

$$
x=\left(x_{1}, y_{1}\right)\left(x_{2}, y_{2}\right) \cdots\left(x_{n}, y_{n}\right) \neq 1 .
$$

Let $H=\left\langle x_{1}, y_{1}, \ldots, x_{n}, y_{n}\right\rangle$; then $x \in H^{\prime}$ and $H$ is finite since $G$ is locally finite. If $C=H \cap P$, then $C$ is a finite normal $p$-subgroup of $G$ since $P$ is central. It suffices to consider $G / C$, or equivalently we can assume $H \cap P=1$. $G^{\prime}$ is not a $p$-group, so $K$ is finite by $[\operatorname{Pas} 97$, Theorem 1.1]. Let $J=J(K[H])$ and write $K[H] / J=\bigoplus \sum_{i} M_{n_{i}}\left(F_{i}\right)$ where $F_{i}$ are fields since $K$ is finite. If all $n_{i}=1$, then $K[H] / J$ is commutative and $x+J=1+J$. Since $J$ is nil, we get that $x$ is a $p$-element, a contradiction. Therefore, there exists some $n_{j} \geq 2$. Since finite fields are perfect, by Wedderburn's Principal Theorem [Row91, Theorem 2.5.37], $K[H]$ contains a copy of $K[H] / J$ and hence it contains a copy of $M_{2}(K)$. Note that $P \times H \cong P H$ since $P$ is central and $H \cap P=1$. Thus we have

$$
\begin{aligned}
M_{2}(K[P]) \cong K[P] \otimes_{K} M_{2}(K) \hookrightarrow K[P] \otimes_{K} K[H] & \\
& \cong K[P \times H] \cong K[P H] \subseteq K[G] .
\end{aligned}
$$


Since $U(K[G])$ satisfies $w=1, U\left(M_{2}(K[P])\right)$ also satisfies $w=1$. If $y$ is any element in $P$, then $1-y$ is nilpotent since $P$ is a $p$-group. Let $a=\left(\begin{array}{cc}0 & 1-y \\ 0 & 0\end{array}\right)$, $b=\left(\begin{array}{ll}0 & 0 \\ 1 & 0\end{array}\right)$. Then $a, b \in M_{2}(K[P])$ and $a b=\left(\begin{array}{cc}1-y & 0 \\ 0 & 0\end{array}\right)$ is nilpotent since $1-y$ is. Lemma 2.2 now implies that $(a b)^{d}=0$. Fix an integer $k$ so that $p^{k} \geq d$. Then $(a b)^{p^{k}}=0$, so we get $(1-y)^{p^{k}}=0$ and $y^{p^{k}}=1$. Hence $P$ has finite period dividing $p^{k}$. This completes the proof.

Lemma 2.10. $1 \Rightarrow 2$.

Proof. [Pas85, Corollary 5.3.10] and [Liu, Theorem 1.1] imply that $G$ has a normal $p$-abelian subgroup of finite index.

If $G^{\prime}$ is a $p$-group, then Lemma 2.8 implies that $G^{\prime}$ has finite period.

If $G^{\prime}$ is not a $p$-group, [Pas97, Theorem 1.1] implies that $K$ must be finite. Since $G$ is a torsion group, Lemma 2.7 and 2.9 imply that the whole group $G$ has finite period.

\section{REFERENCES}

[Coe82] Sônia P. Coelho, Group rings with units of bounded exponent over the center, Can. J. Math. 34 (1982), no. 6, 1349-1364. MR 84c:16014

[GJV94] A. Giambruno, E. Jespers, and A. Valenti, Group identities on units of rings, Arch. Math. (Basel) 63 (1994), no. 4, 291-296. MR 95h:16044

[GSV97] A. Giambruno, S. Sehgal, and A. Valenti, Group algebras whose units satisfy a group identity, Proc. Amer. Math. Soc. 125 (1997), no. 3, 629-634. MR 97f:16055

[Liu] Chia-Hsin Liu, Group algebras with units satisfying a group identity, Proc. Amer. Math. Soc. (this issue) CMP 98:03

[Pas85] D. S. Passman, The algebraic structure of group rings, Robert E. Krieger, Malabar, 1985. MR 86j: 16001

[Pas97] D. S. Passman, Group algebras whose units satisfy a group identity II, Proc. Amer. Math. Soc. 125 (1997), no. 3, 657-662. CMP 97:05

[Row91] L. H. Rowen, Ring theory, Academic Press, San Diego, 1991. MR 94e:16001

Department of Mathematics, University of Wisconsin-Madison, Madison, Wisconsin 53706

E-mail address: chliu@math.wisc.edu

E-mail address: passman@math.wisc.edu 